Pulsar Astronomy -- 2000 and Beyond

ASP Conference Series, Vol. 202, 2000

M. Kramer, N. Wex, and R. Wielebinski, eds.

\title{
Are Pulsars Bare Strange Stars?
}

\author{
R. X. Xu, G. J. Qiao, and B. Zhang \\ CAS-PKU joint Beijing Astrophysical Center and Department of \\ Astronomy, Peking University, Beijing 100781, China
}

\begin{abstract}
.
It is believed that pulsars are neutron stars or strange stars with crusts. However we suggest here that pulsars may be bare strange stars (i.e., strange stars without crust). Due to rapid rotation and strong emission, young strange stars produced in supernova explosions should be bare when they act as radio pulsars. Because of strong magnetic field, two polar-crusts would shield the polar caps of an accreting strange star. Such a suggestion can be checked by further observations.
\end{abstract}

Two greatest theories (i.e., gravity and quantum) developed in this century resulted in the formation of the theory of compact stars. One of such kind of stars observed are pulsars that were discovered by radio astronomers in $1960 \mathrm{~s}$. More observations later in $X$-ray and $\gamma$-ray bands confirmed the existence of pulsars in the nature: a kind of celestial bodies with masses $\sim 1 M_{\odot}$, radius $\sim 10 \mathrm{~km}$, and magnetic fields $\sim 10^{12}$ gausses. Such objects, named as pulsars, are popularly thought to be neutron stars soon after the discoveries.

Owing to the development of the hadronic quark model in 1960s and 1970s, it is conjectured that strange quark matter (SQM), composed of nearly equal numbers of up, down, and strange quarks, may be an absolutely stable 'hadron' of strong interaction confined states. If this assumption is true, strange star ${ }^{1}$ is in the nature, which should be a ground state of neutron star. Hence, radio pulsars survived from supernovae might be strange stars ${ }^{1}$.

It is addressed that a strange quark core is surrounded by a normal matter crust in the conventional strange star scenario for radio pulsar ${ }^{1}$. However, bare strange stars (BSS) can also well act as radio pulsars ${ }^{2}$. If pulsars are BSSs, 1 , some problems in pulsar emission mechanism can be settled ${ }^{2,3}, 2$, strange stars and neutron stars could easily be distinguished in observation.

Pulsars produced after supernovae: bare strange stars? The process of phase transition from neutron matter to SQM can cure the imperfection in the core-collapse supernova paradigm ${ }^{4}$. For progenitors with negligible effect of rotation, additional neutrino emission by forming a strange star can sufficiently enhance the power of neutrino energy deposition behind the stalled shock, thus conduce towards a successful explosion and its enough energy. For progenitors with rapidly rotating inner core, neutron stars might be formed as semifinished products after supernovae. Such a neutron star may be finally phase-converted to a strange star when it spins down, and a $\gamma$-ray burst may appear simultaneously ${ }^{4}$.

There are two reasons for us to inevitably suggest that a strange star as a radio pulsar should be bare. First, the mass ejection rate from an envelope of a 
strange star is very high soon after a supernova, therefore it is natural to expect that the quark surface of a very young strange star is nearly (or completely) bare $^{5}$. The second one is the rapid rotation of newborn strange star. Only when the rotation of a strange star is slowed down enough, would the accretion onto the surface be possible. The conditions for a possible accretion are $r_{\mathrm{m}}<r_{\mathrm{c}}<r_{\mathrm{l}}$. Substituting the radius of magnetosphere $r_{\mathrm{m}}$, the co-rotation radius $r_{\mathrm{c}}$, and the radius of light cylinder $r_{1}$, we find $P_{1}>0.7 M_{8}^{-3 / 7}$ for typical pulsar values, where $\dot{M}_{8}$ is accretion rate in $10^{-8} M_{\odot} \cdot \mathrm{yr}^{-1}$. For solitary strange stars, $\dot{M}_{8}$ is very small because of very strong ejection around the star. If $\dot{M}_{8}=0.01$, only strange stars with periods greater than $5 \mathrm{~s}$ can accrete surrounding matter.

As $s$ quark is a little more massive than that of $u$ and $d$ quarks, there are a few electrons in the chemical equilibrium of SQM in order to keep the matter neutral. The electromagnetic force participated in makes the structure of strange quark matter more interesting and attractive. It is found ${ }^{6}$ that the number density of electrons above quark surface $n_{\mathrm{e}} \sim \frac{9.49 \times 10^{35}}{\left(1.2 z_{11}+4\right)^{3}} \mathrm{~cm}^{-3}$, and the electric field $E \sim \frac{7.18 \times 10^{18}}{\left(1.2 z_{11}+4\right)^{2}} \mathrm{~V} \mathrm{~cm}^{-1}$, where $z$, in $10^{-11} \mathrm{~cm}$, is a measured height above the surface. These analytical results are valuable if we are concerned about physical processes near the quark surfaces of strange stars.

An accreting pulsar: with two polar-crusts? As for strange stars in binaries, they could be accretion powered $X$-ray sources with two polar-crusts ${ }^{2}$. In some cases, the intermittent phase transition in polar-crust could result in a burst process when one of the crusts is heavier than that the Coulomb force can support. GRO J1744-28 is an ideal example for studying such process. The observational phase-lag in GRO J1744-28 may be a strong evidence of the electric gap between strange quark matter and polar-crust. The bursting can cause matter to enough expand and to drag matter to latter phase. Such phaselag can be calculated to be $\sim 60^{\circ}$ if an expanded crust is $\sim 1 \mathrm{~km}$.

Conclusion We suggested that radio pulsars might be bare strange stars, and that accreting strange stars have two polar-crusts. Bare strange stars and neutron stars are distinguishable in observation because their surfaces differ in many ways, such as the electrodynamics, the thermodynamics, and the composition (see, Xu, Qiao \& Zhang in this peroceding). Detailed research on this topic should be interesting and necessary.

We thank Mr. B.H. Hong, Dr. J.L. Han, and other members in our pulsar group. This work is supported by NSFC (No. 19803001, N0.19910211260-570A03), by the Climbing project of China, by Doctoral Program Foundation of Institution of Higher Education in China and by the Youth Foundation of PKU.

\section{References}

(1)Alcock, C., Farhi, E., \& Olinto, A. 1986, ApJ, 310, 261

(2)Xu, R.X., \& Qiao, G.J. 1998, Chin Phys Lett, 15, 934

(3)Xu, R.X., Qiao, G. J., \& Zhang, B. 1999, ApJ, 522, L109

(4)Xu, R.X. et al. 1999, in K.S. Cheng (eds) Pacific Rim Conf. on Stellar Astrophysics, Kluwer Publishers, in press

(5)Usov, V.V., 1998, Phys Rev Lett, 80, 230

(6)Xu, R.X., \& Qiao, G.J. 1999, Chin Phys Lett, 16, 778 